\title{
A TWO ARMED BANDIT TYPE PROBLEM REVISITED
}

\author{
Gilles PAGÈS ${ }^{1}$
}

\begin{abstract}
In Benaïm and Ben Arous (2003) is solved a multi-armed bandit problem arising in the theory of learning in games. We propose a short and elementary proof of this result based on a variant of the Kronecker lemma.
\end{abstract}

Mathematics Subject Classification. 91A20, 91A12, 60F99.

Received December 10, 2004. Revised April 29, 2005.

In [2] a multi-armed bandit problem is addressed and investigated by Benaïm and Ben Arous. Let $f_{0}, \ldots, f_{d}$ denote $d+1$ real-valued continuous functions defined on $[0,1]^{d+1}$. Given a sequence $x=\left(x_{n}\right)_{n \geq 1} \in\{0, \ldots, d\}^{\mathbb{N}^{*}}$ (the strategy), set for every $n \geq 1$

$$
\bar{x}_{n}:=\left(\bar{x}_{n}^{0}, \bar{x}_{n}^{1}, \ldots, \bar{x}_{n}^{d}\right) \quad \text { with } \quad \bar{x}_{n}^{i}:=\frac{1}{n} \sum_{k=1}^{n} \mathbf{1}_{\left\{x_{k}=i\right\}}, \quad i=0, \ldots, d,
$$

and

$$
Q(x)=\liminf _{n \rightarrow+\infty} \frac{1}{n} \sum_{k=0}^{n-1} f_{x_{k+1}}\left(\bar{x}_{k}\right) .
$$

$\left(\bar{x}_{0}:=\left(\bar{x}_{0}^{0}, \bar{x}_{0}^{1}, \ldots, \bar{x}_{0}^{d}\right) \in[0,1]^{d+1}, \bar{x}_{0}^{0}+\cdots+\bar{x}_{0}^{d}=1\right.$, is a starting distribution). Imagine $d+1$ players enrolled in a cooperative/competitive game with the following simple rules: if player $i \in\{0, \ldots, d\}$ plays at time $n$ he is rewarded by $f_{i}\left(\bar{x}_{n}\right)$, otherwise he gets nothing; only one player can play at any given time. Then the sequence $x$ is a playing strategy adopted by the group of players and $Q(x)$ is the global worst cumulative payoff rate of the strategy $x$ for the whole community of players (regardless of the cumulative payoff rate of each player). This interpretation slightly differs from that proposed in [2] where a single player is considered. This player has the choice among $d+1$ "arms" at every time $n$ with a reward $f_{i}\left(\bar{x}_{n}\right)$ when choosing "arm" $i$. We adopt the first one in view of our illustration.

In [2] an answer (see Th. 1 below) is provided to the following question

What are the good strategies (for the group)?

The authors rely on some recent tools developed in stochastic approximation theory (see e.g. [1]). The aim of this note is to provide an elementary and shorter proof based on a slight improvement of the Kronecker lemma. As an illustration, we emphasize that in such a game a greedy strategy is usually not optimal, even for the "individual winner".

Keywords and phrases. Two-armed bandit problem, Kronecker lemma, learning theory, stochastic fictitious play.

1 Laboratoire de Probabilités et Modèles Aléatoires, UMR 7599, Université Paris 6, case 188, 4, place Jussieu, 75252 Paris Cedex 5, France; gpa@ccr.jussieu.fr 
Let $\mathcal{S}_{d}:=\left\{v=\left(v^{1}, \ldots, v^{d}\right) \in[0,1]^{d}, \sum_{i=1}^{d} v_{i} \leq 1\right\}$ and $\mathcal{P}_{d+1}:=\left\{u=\left(u^{0}, u^{1}, \ldots, u^{d}\right) \in[0,1]^{d+1}, \sum_{i=1}^{d+1} u_{i}=\right.$ $1\}$. Furthermore, for notational convenience, set

$$
\begin{aligned}
\forall v \in \mathcal{S}_{d}, \tilde{v} & :=\left(1-\sum_{i=1}^{d} v^{i}, v^{1}, \ldots, v^{d}\right) \in \mathcal{P}_{d+1}, \\
\forall u \in \mathcal{P}_{d+1},{ }^{\sigma} u & :=\left(u^{1}, \ldots, u^{d}\right) \in \mathcal{S}_{d} .
\end{aligned}
$$

The canonical inner product on $\mathbb{R}^{d}$ will be denoted by $(v \mid w)=\sum_{i=1}^{d} v^{i} w^{i}$. The interior of a subset $A$ of $\mathbb{R}^{d}$ will be denoted by $\AA$. For a sequence $u=\left(u_{n}\right)_{n \geq 0}, \Delta u_{n}:=u_{n}-u_{n-1}, n \geq 1$.

The main result is the following theorem (first established in [2]).

Theorem 1. Assume there is a continuous function $\Phi: \mathcal{S}_{d} \rightarrow \mathbb{R}$, continuously differentiable on $\stackrel{\circ}{\mathcal{S}}_{d}$, having a continuous extension of its gradient $\nabla \Phi$ on $\mathcal{S}_{d}$ and satisfying:

$$
\forall v \in \mathcal{S}_{d}, \quad \nabla \Phi(v)=\left(f_{i}(\tilde{v})-f_{0}(\tilde{v})\right)_{1 \leq i \leq d}
$$

Set for every $u \in \mathcal{P}_{d+1}$,

$$
q(u):=\sum_{i=0}^{d+1} u^{i} f_{i}(u)
$$

and $Q^{*}:=\max \left\{q(u), u \in \mathcal{P}_{d+1}\right\}$. Then, for every strategy $x \in\{0,1, \ldots, d\}^{\mathbb{N}^{*}}$,

$$
Q(x) \leq Q^{*}
$$

Furthermore, for any strategy $x$ such that $\bar{x}_{n} \rightarrow \bar{x}_{\infty}$,

$$
\frac{1}{n} \sum_{k=0}^{n-1} f_{x_{k+1}}\left(\bar{x}_{k}\right) \rightarrow q\left(\bar{x}_{\infty}\right) \quad \text { as } \quad n \rightarrow \infty \quad\left(\text { so that } Q(x)=q\left(\bar{x}_{\infty}\right)\right) .
$$

In particular there is no better strategy than choosing the player at random according to an i.i.d. "Bernouilli strategy" with parameter $\bar{x}^{*} \in \operatorname{argmax} q$.

The key of the proof is the following slight extension of the Kronecker lemma.

Lemma 1 ("à la Kronecker" lemma). Let $\left(b_{n}\right)_{n \geq 1}$ be a nondecreasing sequence of positive real numbers converging to $+\infty$ and let $\left(a_{n}\right)_{n \geq 1}$ be a sequence of real numbers. Then

$$
\liminf _{n \rightarrow+\infty} \sum_{k=1}^{n} \frac{a_{k}}{b_{k}} \in \mathbb{R} \Longrightarrow \liminf _{n \rightarrow+\infty} \frac{1}{b_{n}} \sum_{k=1}^{n} a_{k} \leq 0 .
$$

Proof. Set $C_{n}=\sum_{k=1}^{n} \frac{a_{k}}{b_{k}}, n \geq 1$, and $C_{0}=0$ so that $a_{n}=b_{n} \Delta C_{n}$. As a consequence, an Abel transform yields

$$
\begin{aligned}
\frac{1}{b_{n}} \sum_{k=1}^{n} a_{k} & =\frac{1}{b_{n}} \sum_{k=1}^{n} b_{k} \Delta C_{k}=\frac{1}{b_{n}}\left(b_{n} C_{n}-\sum_{k=1}^{n} C_{k-1} \Delta b_{k}\right) \\
& =C_{n}-\frac{1}{b_{n}} \sum_{k=1}^{n} C_{k-1} \Delta b_{k} .
\end{aligned}
$$


Now, $\liminf _{n \rightarrow+\infty} C_{n}$ being finite, for every $\varepsilon>0$, there is an integer $n_{\varepsilon}$ such that for every $k \geq n_{\varepsilon}, C_{k} \geq \liminf _{n \rightarrow+\infty} C_{n}-\varepsilon$. Hence

$$
\frac{1}{b_{n}} \sum_{k=1}^{n} C_{k-1} \Delta b_{k} \geq \frac{1}{b_{n}} \sum_{k=1}^{n_{\varepsilon}} C_{k-1} \Delta b_{k}+\frac{b_{n}-b_{n_{\varepsilon}}}{b_{n}}\left(\liminf _{k} C_{k}-\varepsilon\right)
$$

Consequently, $\liminf _{n \rightarrow+\infty} C_{n}$ being finite, one concludes that for every $\varepsilon>0$,

$$
\liminf _{n \rightarrow+\infty} \frac{1}{b_{n}} \sum_{k=1}^{n} a_{k} \leq \liminf _{n \rightarrow+\infty} C_{n}-0-1 \times\left(\liminf _{k \rightarrow+\infty} C_{k}-\varepsilon\right)=\varepsilon
$$

Proof of Theorem 1. First note that for every $u=\left(u^{0}, u^{1}, \ldots, u^{d}\right) \in \mathcal{P}_{d+1}$,

$$
q(u):=\sum_{i=0}^{d+1} u^{i} f_{i}(u)=f_{0}(u)+\sum_{i=1}^{d} u^{i}\left(f_{i}(u)-f_{0}(u)\right)
$$

so that

$$
Q^{*}=\sup _{v \in \mathcal{S}_{d}}\left\{f_{0}(\tilde{v})+\sum_{i=1}^{d} v^{i}\left(f_{i}(\tilde{v})-f_{0}(\tilde{v})\right)\right\}=\sup _{v \in \mathcal{S}_{d}}\left\{f_{0}(\tilde{v})+(v \mid \nabla \Phi(v))\right\} .
$$

Now, for every $k \geq 0$,

$$
\begin{aligned}
f_{x_{k+1}}\left(\bar{x}_{k}\right)-q\left(\bar{x}_{k}\right) & =\sum_{i=0}^{d}\left(f_{i}\left(\bar{x}_{k}\right) \mathbf{1}_{\left\{x_{k+1}=i\right\}}-\bar{x}_{k}^{i} f_{i}\left(\bar{x}_{k}\right)\right)=\sum_{i=0}^{d} f_{i}\left(\bar{x}_{k}\right)\left(\mathbf{1}_{\left\{x_{k+1}=i\right\}}-\bar{x}_{k}^{i}\right) \\
& =\sum_{i=0}^{d} f_{i}\left(\bar{x}_{k}\right)(k+1) \Delta \bar{x}_{k+1}^{i} \\
& =(k+1) \sum_{i=1}^{d}\left(f_{i}\left(\bar{x}_{k}\right)-f_{0}\left(\bar{x}_{k}\right)\right) \Delta \bar{x}_{k+1}^{i}
\end{aligned}
$$

The last equality reads using Assumption (2) and notation (1),

$$
f_{x_{k+1}}\left(\bar{x}_{k}\right)-q\left(\bar{x}_{k}\right)=(k+1)\left(\nabla \Phi\left({ }^{\sigma} \bar{x}_{k}\right) \mid \Delta^{\sigma} \bar{x}_{k+1}\right) .
$$

Consequently, by the fundamental formula of calculus applied to $\Phi$ on $\left({ }^{\sigma} \bar{x}_{k},{ }^{\sigma} \bar{x}_{k+1}\right) \subset \stackrel{\circ}{\mathcal{S}}_{d}$,

$$
\begin{aligned}
\frac{1}{n} \sum_{k=0}^{n-1} f_{x_{k+1}}\left(\bar{x}_{k}\right)-q\left(\bar{x}_{k}\right) & =\frac{1}{n} \sum_{k=0}^{n-1}(k+1)\left(\Phi\left({ }^{\sigma} \bar{x}_{k+1}\right)-\Phi\left({ }^{\sigma} \bar{x}_{k}\right)\right)-R_{n} \\
\text { with } \quad R_{n} & :=\frac{1}{n} \sum_{k=0}^{n-1}\left(\nabla \Phi\left(\xi_{k}\right)-\nabla \Phi\left({ }^{\sigma} \bar{x}_{k}\right) \mid(k+1) \Delta^{\sigma} \bar{x}_{k+1}\right)
\end{aligned}
$$

and $\xi_{k} \in\left({ }^{\sigma} \bar{x}_{k},{ }^{\sigma} \bar{x}_{k+1}\right), k=0, \ldots, n-1$. The fact that $\left|(k+1) \Delta^{\sigma} \bar{x}_{k+1}\right| \leq 1$ implies

$$
\left|R_{n}\right| \leq \frac{1}{n} \sum_{k=0}^{n-1} w\left(\nabla \Phi,\left|\Delta^{\sigma} \bar{x}_{k+1}\right|\right)
$$


where $w(g, \delta)$ denotes the uniform continuity $\delta$-modulus of a function $g$. One derives from the uniform continuity of $\nabla \Phi$ on the compact set $\mathcal{S}_{d}$ that

$$
R_{n} \rightarrow 0 \quad \text { as } \quad n \rightarrow+\infty
$$

Finally, the continuous function $\Phi$ being bounded on the compact set $\mathcal{S}_{d}$, the partial sums

$$
\sum_{k=0}^{n-1} \Phi\left({ }^{\sigma} \bar{x}_{k+1}\right)-\Phi\left({ }^{\sigma} \bar{x}_{k}\right)=\Phi\left({ }^{\sigma} \bar{x}_{n+1}\right)-\Phi\left({ }^{\sigma} \bar{x}_{0}\right)
$$

remain bounded as $n$ goes to infinity. Lemma 1 then implies that

$$
\liminf _{n \rightarrow+\infty} \frac{1}{n} \sum_{k=0}^{n-1}(k+1)\left(\Phi\left({ }^{\sigma} \bar{x}_{k+1}\right)-\Phi\left({ }^{\sigma} \bar{x}_{k}\right)\right) \leq 0
$$

One concludes by noting that on one hand

$$
\limsup _{n \rightarrow+\infty} \frac{1}{n} \sum_{k=0}^{n-1} q\left(\bar{x}_{k}\right) \leq Q^{*}=\sup _{\mathcal{P}_{d+1}} q
$$

and that, on the other hand, the function $q$ being continuous,

$$
\lim _{n \rightarrow+\infty} \frac{1}{n} \sum_{k=0}^{n-1} q\left(\bar{x}_{k}\right)=q\left(x^{*}\right) \quad \text { as soon as } \quad \bar{x}_{n} \rightarrow x^{*} .
$$

Corollary 1. When $d+1=2$ (two players), Assumption (2) is satisfied as soon as $f_{0}$ and $f_{1}$ are continuous on $\mathcal{P}_{2}$ and then the conclusions of Theorem 1 hold true.

Proof. This follows from the obvious fact that the continuous function $u^{1} \mapsto f_{1}\left(1-u^{1}, u^{1}\right)-f_{0}\left(1-u^{1}, u^{1}\right)$ on $[0,1]$ has an antiderivative.

\section{Further comments:}

- If one considers a slightly more general game in which some weighted strategies are allowed, the final result is not modified in any way provided the weight sequence satisfies a very light assumption. Namely, assume that at time $n$ the reward is

$$
\Delta_{n+1} f_{x_{n+1}}\left(\bar{x}_{n}\right) \quad \text { instead of } \quad f_{x_{n+1}}\left(\bar{x}_{n}\right)
$$

where the weight sequence $\Delta=\left(\Delta_{n}\right)_{n \geq 1}$ satisfies

$$
\Delta_{n} \geq 0, n \geq 1, \quad S_{n}=\sum_{k=1}^{n} \Delta_{k} \rightarrow+\infty, \quad \frac{\Delta_{n}}{S_{n}} \rightarrow 0 \text { as } n \rightarrow \infty
$$

then the quantities $\bar{x}_{0}^{\Delta} \in \mathcal{P}_{d+1}, \bar{x}_{n}^{\Delta}:=\left(\bar{x}_{n}^{\Delta, 0}, \ldots, \bar{x}_{n}^{\Delta, d}\right)$ with $\bar{x}_{n}^{\Delta, i}=\frac{1}{S_{n}} \sum_{k=1}^{n} \Delta_{k} \mathbf{1}_{\left\{x_{k}=i\right\}}, i=0, \ldots, d, n \geq 1$, and $Q^{\Delta}(x)=\liminf _{n \rightarrow+\infty} \frac{1}{S_{n}} \sum_{k=0}^{n-1} \Delta_{k+1} f_{x_{k+1}}\left(\bar{x}_{k}^{\Delta}\right)$ satisfy all the conclusions of Theorem 1 mutatis mutandis.

- Several applications of Theorem 1 to the theory of learning in games and to stochastic fictitious play are extensively investigated in [2] which we refer to for all these aspects. As far as we are concerned we will simply make a remark about some "natural" strategies which illustrates the theorem in an elementary way.

In the reward function at time $k$, i.e. $f_{x_{k}}\left(\bar{x}_{k-1}\right), x_{k}$ represents the competitive term ("who will play?") and $\bar{x}_{k-1}$ represents a cooperative term (everybody's past behaviour has influence on everybody's reward). 
This cooperative/competitive antagonism induces that in such a game a greedy competitive strategy is usually not optimal (when the players do not play a symmetric role). Let us be more specific. Assume for the sake of simplicity that $d+1=2$ (two players). Then one may consider without loss of generality that $\bar{x}_{n}={ }^{\sigma} \bar{x}_{n} i . e$. that $\bar{x}_{n}$ is a $[0,1]$-valued real number. A greedy competitive strategy is defined by

$$
\text { player } \left.1 \text { plays at time } n \text { (i.e. } x_{n}=1\right) \quad \text { iff } \quad f_{1}\left(\bar{x}_{n-1}\right) \geq f_{0}\left(\bar{x}_{n-1}\right)
$$

i.e. the player with the highest reward is nominated to play. Then, for every $n \geq 1$,

$$
f_{x_{n}}\left(\bar{x}_{n-1}\right)=\max \left(f_{0}\left(\bar{x}_{n-1}\right), f_{1}\left(\bar{x}_{n-1}\right)\right)
$$

and it is clear that

$$
f_{x_{n}}\left(\bar{x}_{n-1}\right)-q\left(\bar{x}_{n-1}\right)=\max \left(f_{0}\left(\bar{x}_{n-1}\right), f_{1}\left(\bar{x}_{n-1}\right)\right)-q\left(\bar{x}_{n-1}\right)=: \varphi\left(\bar{x}_{n-1}\right) \geq 0 .
$$

On the other hand, the proof of Theorem 1 implies that

$$
\liminf _{n \rightarrow+\infty} \frac{1}{n} \sum_{k=0}^{n-1} \varphi\left(\bar{x}_{k}\right) \leq 0 .
$$

Hence, there is at least one weak limiting distribution $\bar{\mu}_{\infty}$ of the sequence of empirical measures $\bar{\mu}_{n}:=$ $\frac{1}{n} \sum_{0 \leq k \leq n-1} \delta_{\bar{x}_{k}}$ on the compact interval [0,1] which is supported by the closed set $\{\varphi=0\} \subset\{0,1\} \cup\left\{f_{0}=f_{1}\right\}$; on the other hand $\operatorname{supp}\left(\mu_{\infty}\right)$ is contained in the set $\overline{\mathcal{X}}_{\infty}$ of the limiting values of the sequence $\left(\bar{x}_{n}\right)$ itself (in fact $\overline{\mathcal{X}}_{\infty}$ is an interval since $\left(\bar{x}_{n}\right)_{n}$ is bounded and $\left.\bar{x}_{n+1}-\bar{x}_{n} \rightarrow 0\right)$. Hence $\overline{\mathcal{X}}_{\infty} \cap\left(\{0,1\} \cup\left\{f_{0}=f_{1}\right\}\right) \neq \emptyset$.

If the greedy strategy $\left(\bar{x}_{n}\right)_{n}$ is optimal then $\operatorname{dist}\left(\bar{x}_{n}, \operatorname{argmax} q\right) \rightarrow 0$ as $n \rightarrow \infty$ i.e. $\overline{\mathcal{X}}_{\infty} \subset \operatorname{argmax} q$. Consequently if

$$
\operatorname{argmax} q \cap\left(\{0,1\} \cup\left\{f_{0}=f_{1}\right\}\right)=\emptyset
$$

then the purely competitive strategy is never optimal for the group of two players.

Let us be more specific on the following example: set for two positive parameters $a \neq b$

$$
f_{0}(x):=a x \quad \text { and } \quad f_{1}(x):=b(1-x), \quad x \in[0,1] .
$$

Then one checks that

$$
\operatorname{argmax} q=\{1 / 2\} \quad \text { and } \quad f_{0}(1 / 2) \neq f_{1}(1 / 2) .
$$

One first shows that the greedy strategy $x=\left(x_{n}\right)_{n \geq 1}$ defined by (3) satisfies

$$
\bar{x}_{n} \rightarrow \frac{b}{a+b} \quad \text { and } \quad Q(x)=\frac{a b}{a+b} \quad \text { as } \quad n \rightarrow \infty .
$$

On the other hand, any optimal (cooperative) strategy (like the i.i.d. Bernoulli(1/2) one) yields an asymptotic (relative) global payoff rate

$$
Q^{*}=\max _{[0,1]} q=\frac{a+b}{4} .
$$

Note that $Q^{*}>\frac{a b}{a+b}$ since $a \neq b$. (When $a=b$ the greedy strategy becomes optimal.)

Now, if one looks at the individual performances (i.e. $\left.\lim _{n} \frac{1}{n} \sum_{0 \leq k \leq n-1} f_{i}\left(\bar{x}_{k}\right) \mathbf{1}_{\left\{x_{k+1}=i\right\}}, i=0,1\right)$ of both players when the greedy strategy is played, one checks that:

- the "winner" of the game is player 1 if $b>a$ and player 0 if $a>b$,

- the asymptotic (relative) payoff rate of the winner is equal to $\frac{a b \max (a, b)}{(a+b)^{2}}$ (and $\frac{a b \min (a, b)}{(a+b)^{2}}$ for the "looser"). 
If an optimal cooperative strategy is adopted by the players the "winner" remains the same but with an asymptotic payoff rate equal to $\frac{\max (a, b)}{4}$ (the "looser" gets $\frac{\min (a, b)}{4}$ ). Consequently (when $a \neq b$ ), an optimal cooperative strategy always yields to the winner a strictly higher asymptotic payoff rate than the greedy one. This is also true for the looser.

- A more abstract version of Theorem 1 can be established using the same approach. The finite set $\{0,1, \ldots, d\}$ is replaced by a compact metric set $K, \mathcal{P}_{d+1}$ is replaced by the convex set $\mathcal{P}_{K}$ of probability distributions on $K$ equipped with the weak topology and the continuous function $f: K \times \mathcal{P}_{K} \rightarrow \mathbb{R}$ is still supposed to derive from a potential function in some sense.

\section{REFERENCES}

[1] M. Benaïm, Dynamics of stochastic algorithms, in Séminaire de probabilités XXXIII, J. Azéma et al. Eds., Springer-Verlag, Berlin. Lect. Notes Math. 1708 (1999) 1-68.

[2] M. Benaïm and G. Ben Arous, A two armed bandit type problem. Game Theory 32 (2003) 3-16. 